\title{
Dripping Channel Based Liquid Triboelectric Nanogenerators for Energy Harvesting and Sensing
}

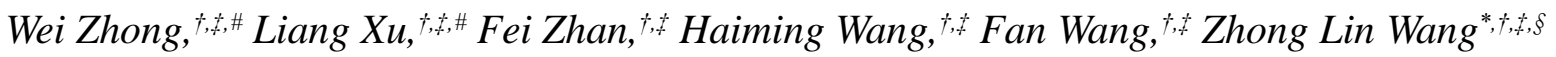

${ }^{\dagger}$ CAS Center for Excellence in Nanoscience, Beijing Key Laboratory of Micro-nano Energy and Sensor, Beijing Institute of Nanoenergy and Nanosystems, Chinese Academy of Sciences, Beijing, 100083, P. R. China

$\$$ School of Nanoscience and Technology, University of Chinese Academy of Sciences, Beijing, 100049, P. R. China

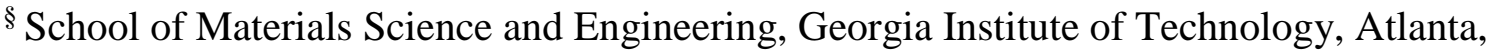
Georgia, 30332, USA

*E-mail: zhong.wang@mse.gatech.edu (Z. L. Wang)

\# These authors contributed equally to this work. 


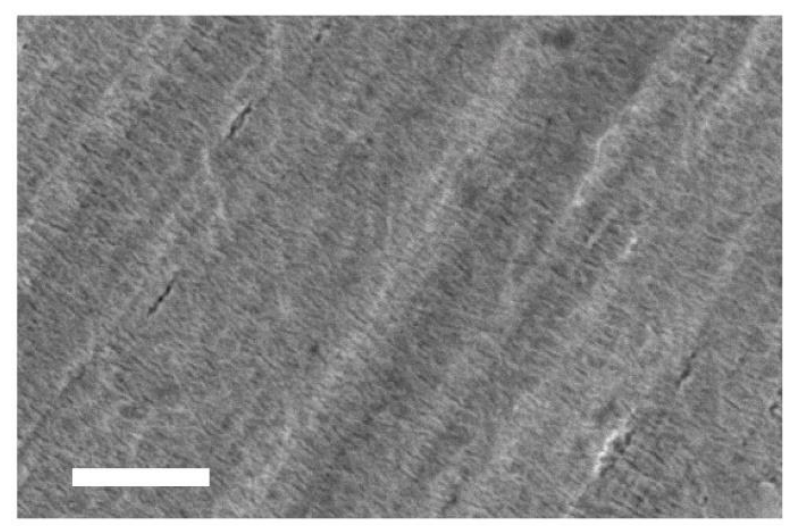

Figure S1. SEM image of the PTFE surface (Scale bar: $3 \mu \mathrm{m}$ ).

(a)

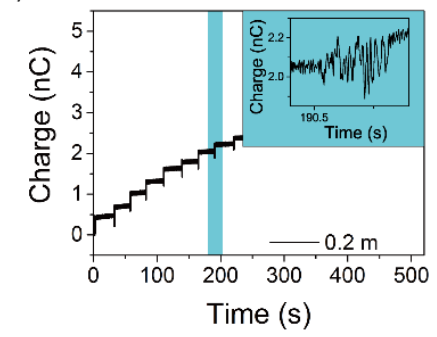

(b)

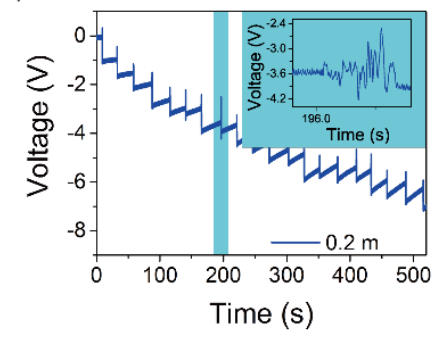

(c)

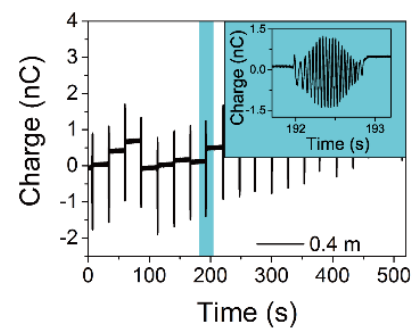

(d)

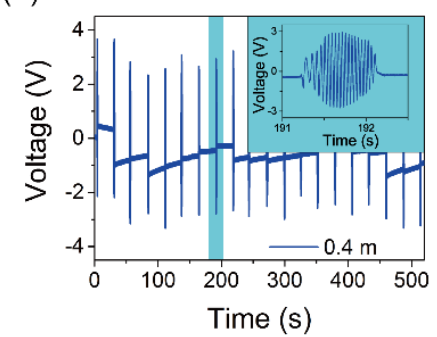

(e)

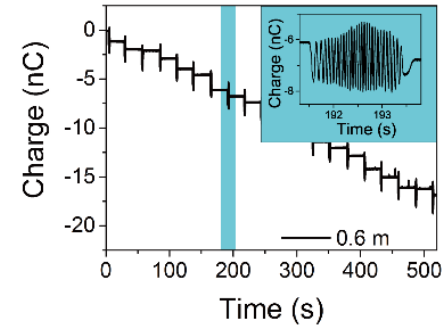

(f)

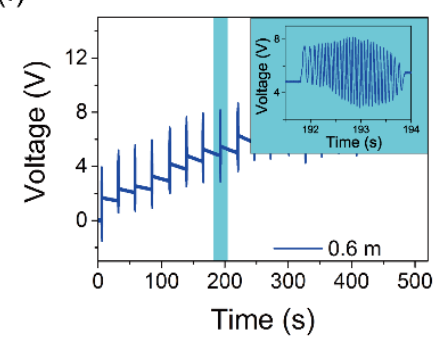

Figure S2. Basic characterization of the unfolded grating-electrode L-TENGs tested with a motor. (a, b) Transferred charges (a) and open-circuit voltage (b) of the $0.2 \mathrm{~m}$ case. (c, d) Transferred charges (c) and open-circuit voltage (d) of the $0.4 \mathrm{~m}$ case. (e, f) Transferred charges (e) and open-circuit voltage (f) of the $0.6 \mathrm{~m}$ case. 


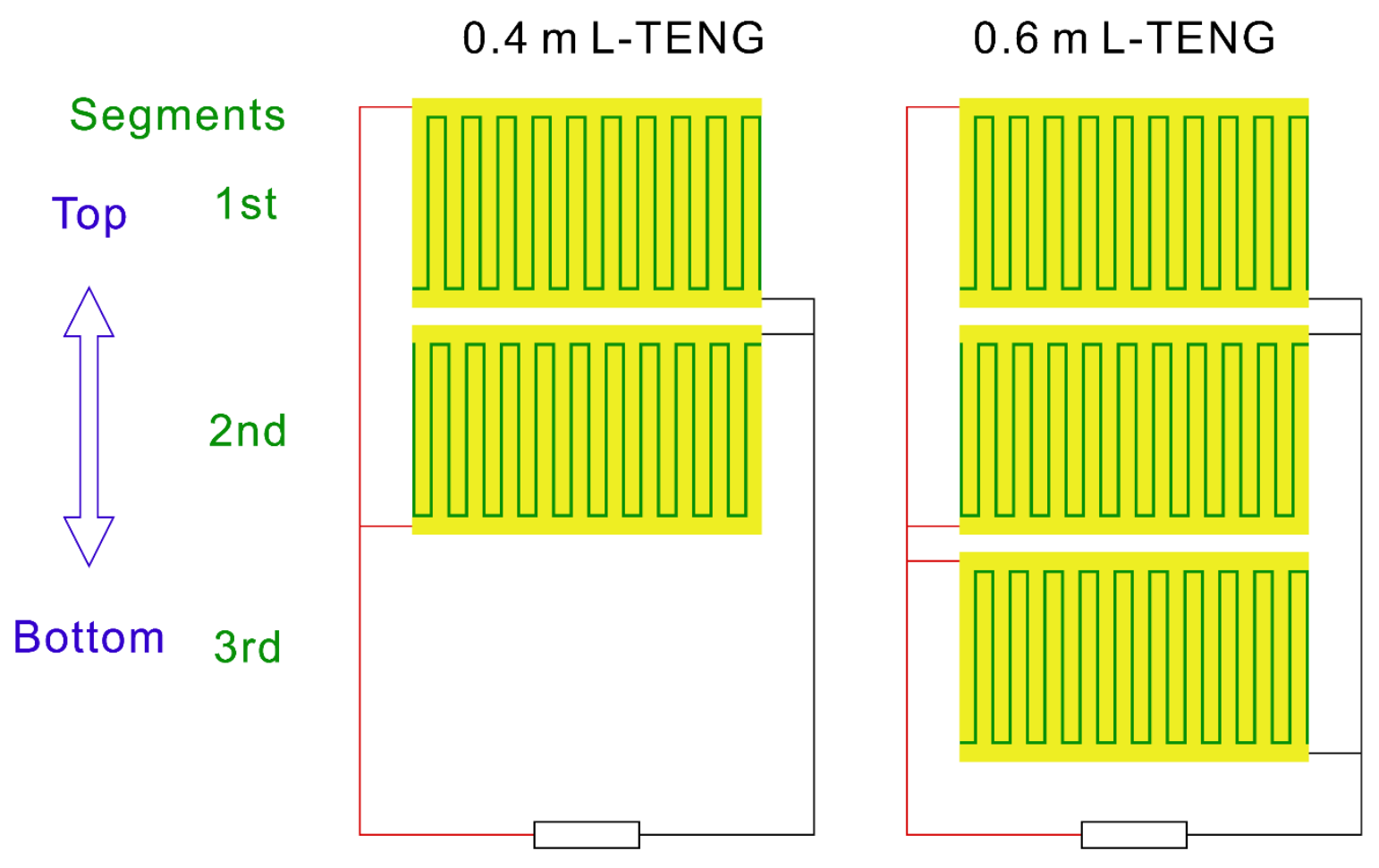

Figure S3. Electrode connections of different segments in the folded $0.4 \mathrm{~m}$ and $0.6 \mathrm{~m}$ grating-electrode L-TENGs. 
(a)

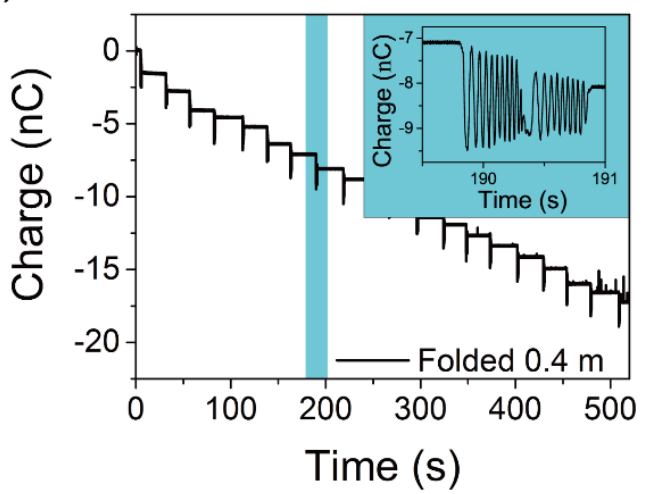

(b)

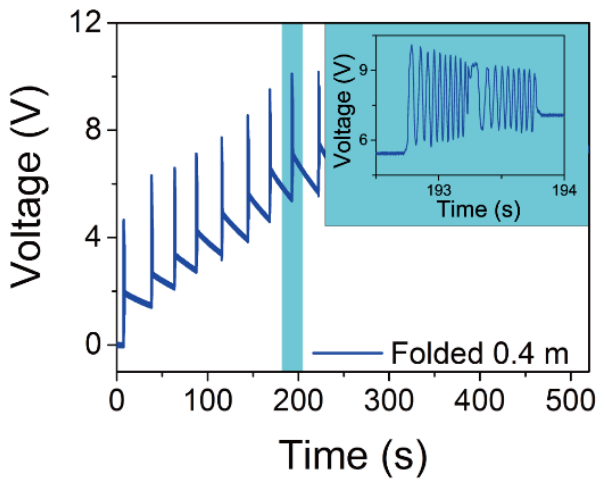

(c)

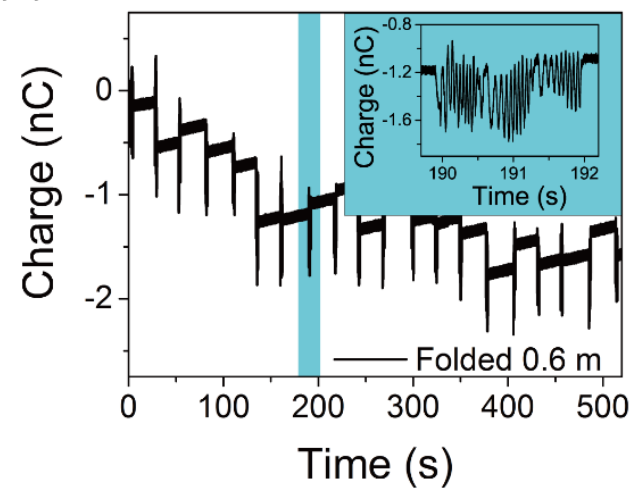

(d)

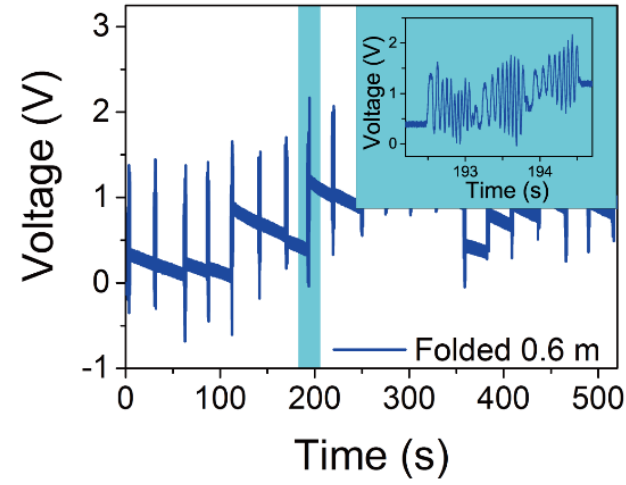

Figure S4. Basic characterization of the folded grating-electrode L-TENGs tested with a motor. (a, b) Transferred charges (a) and open-circuit voltage (b) of the $0.4 \mathrm{~m}$ case. (c, d) Transferred charges (c) and open-circuit voltage (d) of the $0.6 \mathrm{~m}$ case. 
(a)

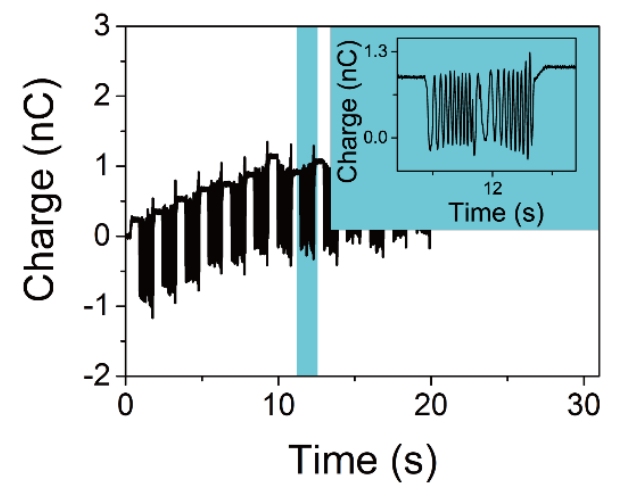

(b)

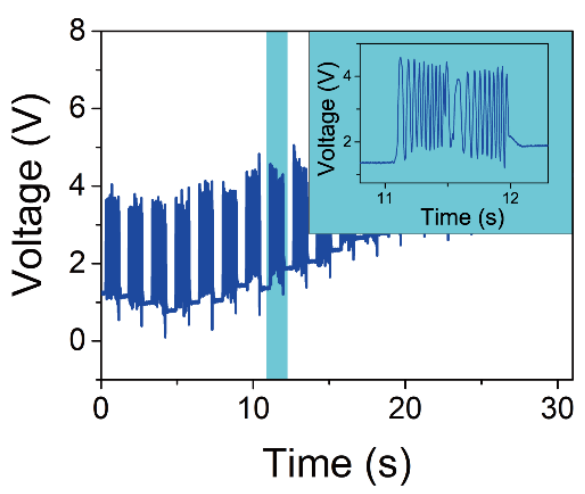

Figure S5. Basic characterization of the folded grating-electrode L-TENGs tested with a funnel. (a, b) Transferred charges (a) and open-circuit voltage (b) of the $0.4 \mathrm{~m}$ case. 